\title{
Uso de exergames de dança no tratamento de doenças neurológicas
}

\author{
Use of dance exergames in the \\ treatment of neurological diseases
}
Uso de exergames de danza en el tratamiento de enfermedades neurológicas

\author{
Vanessa Oliveira Dias ${ }^{1}$, Johseph Paballo Gomes de Souza², Luan \\ Emannuel de Lima Julião ${ }^{3}$, Rodrigo Marcel Valentim da Silva ${ }^{4}$
}

\begin{abstract}
1.Fisioterapeuta, Pós-graduanda em Fisioterapia Neurofuncional na Faculdade Estácio FATERN. Natal-RN, Brasil. https://orcid.org/0000-0002-8333-2195

2.Psicólogo, Mestre em Neuroengenharia, Doutorando em Neurociências no Instituto do Cérebro da Universidade Federal do Rio Grande do Norte (UFRN). Natal-RN, Brasil. https://orcid.org/0000-00026307-0773

3.Graduando em Psicologia no Centro Universitário Maurício de Nassau, Departamento de Psicologia. Natal-RN, Brasil. https://orcid.org/0000-0003-4555-5934

4.Fisioterapeuta, Doutor, Docente do curso de Fisioterapia da Faculdade Estácio FATERN e do Centro Universitário Mauricio de Nassau. Natal-RN, Brasil. https://orcid.org/0000-0002-5859-4599
\end{abstract}

\begin{abstract}
Resumo
Introdução. Nos últimos anos, novas tecnologias como os jogos ativos de videogame foram incorporadas no âmbito da reabilitação. Os jogos de dança promovem a interação direta com o próprio jogo estimulando a atividade física de forma lúdica e vêm se mostrando promissores na terapêutica neurológica. Método. Foi realizada uma busca não sistemática nas bases de dados PubMed (National Library of Medicine National Institutes of Healf), BVS (Biblioteca Virtual em Saúde) e Google Scholar para pesquisa de estudos em português e inglês. Resultados. Os jogos de dança somam os benefícios proporcionados pela prática da dança com as vantagens favorecidas pelo uso de videogames. Com esta pesquisa pôde-se encontrar melhorias físicas, cognitivo-motoras e psicossociais em indivíduos com disfunções neurológicas como sequelas de Acidente vascular cerebral (AVC), doença de Parkinson, Esclerose Múltipla e Doença de Huntington. Conclusão. Os jogos de dança são ferramentas divertidas e prazerosas que proporcionam benefícios em diversos componentes físicos e psicossociais como a neuroplasticidade, cognição, marcha, equilíbrio, sintomas depressivos e de ansiedade em indivíduos com doenças neurológicas. Mais estudos são necessários para maiores esclarecimentos sobre essa temática.
\end{abstract}

Unitermos. Jogos de Vídeo; Dança; Doenças do Sistema Nervoso

\begin{abstract}
Introduction. In recent years, new technologies such as active video game games have been incorporated into the scope of rehabilitation. Dance games promote direct interaction with the game itself, stimulating physical activity in a playful way and have been showing promise in neurological therapy. Method. A non-systematic search was performed in PubMed (National Library of Medicine, National Institutes of Healf), BVS (Virtual Health Library) and Google Scholar databases to search for studies in Portuguese and English. Results. Dance games add the benefits provided by the practice of dance with the advantages favored by the use of video games. With this research it was possible to find physical, cognitive-motor and psychosocial improvements in individuals with neurological disorders such as sequelae of cerebrovascular accident (CVA), Parkinson's disease, Multiple Sclerosis and Huntington's disease. Conclusion. Dance games are fun and pleasurable tools that provide benefits in various physical and psychosocial components such as neuroplasticity, cognition, gait, balance, depressive and anxiety symptoms in individuals with neurological diseases. More studies are needed for further clarification on this topic.
\end{abstract}

Keywords. Video games; Dance; Nervous System Diseases 


\section{Resumen}

Introducción. En los últimos años se han incorporado al ámbito de la rehabilitación nuevas tecnologías como los videojuegos activos. Los juegos de baile promueven la interacción directa con el juego en sí, estimulando la actividad física de forma lúdica y se han mostrado prometedores en la terapia neurológica. Método. Se realizó una búsqueda no sistemática en las bases de datos PubMed (Biblioteca Nacional de Medicina, Institutos Nacionales de Salud), BVS (Biblioteca Virtual de Salud) y Google Scholar para buscar estudios en portugués e inglés. Resultados. Los juegos de danza suman los beneficios que brinda la práctica de la danza con las ventajas que favorece el uso de videojuegos. Con esta investigación fue posible encontrar mejoras físicas, cognitivo-motoras y psicosociales en individuos con trastornos neurológicos como secuelas de accidente cerebrovascular (ACV), enfermedad de Parkinson, esclerosis múltiple y enfermedad de Huntington. Conclusión. Los juegos de baile son herramientas divertidas y placenteras que brindan beneficios en diversos componentes físicos y psicosociales como neuroplasticidad, cognición, marcha, equilibrio, síntomas depresivos y de ansiedad en individuos con enfermedades neurológicas. Se necesitan más estudios para obtener más aclaraciones sobre este tema.

Palabras clave. Videojuegos; Baile; Enfermedades del sistema nervioso

Trabalho realizado na Faculdade Estácio FATERN, Natal-RN, Brasil.

\section{INTRODUÇÃO}

Ao longo da história, a humanidade utilizou a dança como uma de suas principais formas de expressão social, quer sejam por meio de rituais, religiosidade, espetáculos ou estilo de vida, de maneira que ela se faz presente no cotidiano de diversas culturas até hoje. Através dela, se percebe uma maior conexão entre corpo, mente e sentimentos de forma prazerosa e divertida ${ }^{1}$.

A dança é caracterizada pela manifestação do corpo, através de movimentos guiados por um ritmo, logo essa integração entre capacidades físicas e mentais, além das questões sociais, culturais e afetivas envolvidas na dança, possibilitam que ela seja diretamente relacionada com a promoção da saúde e qualidade de vida ${ }^{1}$. 
Com base em todos esses benefícios, a dança vem sendo utilizada na recuperação de problemas tanto de condições naturais, como no processo de envelhecimento ${ }^{2}$, e no tratamento de doenças neurológicas ${ }^{3}$.

No que tange ao processo de envelhecimento, ela é utilizada para minimizar as perdas fisiológicas relacionadas, pois promovem o fortalecimento muscular, melhora da postura, flexibilidade e equilíbrio, além de estimular a oxigenação do cérebro e a cognição ${ }^{1}$. Muitos desses benefícios citados foram também identificados quando utilizados em pessoas com doenças neurológicas ${ }^{3,4}$, o que aponta para a viabilidade do uso desta prática como terapia complementar na reabilitação desse tipo de condição clínica.

Nos últimos anos novas tecnologias foram incorporadas no âmbito da reabilitação. Uma dessas tecnologias foi o exergame. Os exergames são jogos ativos de videogame que promovem a interação direta com o próprio jogo e estimulam a atividade física através da brincadeira ${ }^{5}$. Apesar da maioria dos jogos disponíveis não serem voltados para fins terapêuticos, eles podem ser tão efetivos quanto a reabilitação convencional6.

Sugere-se que são muitas as vantagens do uso dos exergames, incluindo a prevenção da monotonia e do tédio causado por longas sessões de exercícios, aumento da motivação no tratamento e até mesmo a possibilidade de realizar os exercícios fora do ambiente do ambulatório ${ }^{6,7}$.

A utilização de um exergame baseado em jogo de dança é uma estratégia terapêutica promissora que pode 
aliar os benefícios da dança e do exergame e vem se mostrando benéfica tanto em aspectos físicos ${ }^{2}$ quanto psicológicos ${ }^{8}$, inclusive em doenças como Esclerose Múltipla, sequelas de acidente vascular cerebral (AVC) e Doença de Parkinson $^{7-9}$.

Embora existam diversos estudos sobre os benefícios da dança em indivíduos com problemas neurológicos ${ }^{3,4,10,11} \mathrm{e}$ sobre o uso de exergames na reabilitação ${ }^{12-14}$, poucos trabalhos exploram as vantagens da junção dessas duas técnicas ${ }^{7,9}$. Assim, este estudo tem como objetivo discutir sobre o uso dos exergames baseados em dança no tratamento de doenças neurológicas, expondo seus benefícios fisiológicos e psicológicos, riscos e viabilidade da aplicação de acordo com a literatura explorada.

\section{MÉTODO}

Esse artigo trata-se de uma revisão narrativa elaborada através da busca de estudos nas bases de dados: PubMed (National Library of Medicine National Institutes of Healf), BVS (Biblioteca Virtual em Saúde) e Google Scholar. A busca foi realizada no período de outubro de 2020 a junho de 2021, através de títulos, resumos e descritores de assunto com as palavras-chave: "Dance", "Virtual Reality", "Nervous System Diseases", "Multiple Sclerosis", "Parkinson disease", "Stroke", "Huntington disease". Além desses foram utilizadas as palavras-chave: "Exergame", "Active Video Games", "Dance Games", "Just Dance", "Dance Dance Revolution" e "Dance Central" que apesar de não fazerem parte da 
DeCS/MeSH, são de grande relevância para o tema proposto.

A seleção dos artigos foi feita de maneira não sistemática através da leitura do título e do resumo. Foram incluídos ensaios clínicos, estudos de caso, série de casos, estudos piloto ou qualquer estudo de intervenção que abordasse o uso de jogos de dança em amostras com condições clínicas neurológicas, publicados entre 2011 e 2021 nos idiomas português e inglês. Como critério de exclusão, podemos citar que foram excluídos estudos realizados com crianças e adolescentes.

\section{RESULTADOS}

Foram selecionados 10 estudos que corresponderam aos critérios de inclusão e exclusão. Dentre eles, quatro artigos utilizaram os jogos de dança em indivíduos com Doença de Parkinson ${ }^{8,15-17}$, três em pessoas com sequelas de AVC $^{9,18,19}$, um em participantes com doença de Huntington ${ }^{20}$, um em pessoas com Esclerose Múltipla ${ }^{2}$ e um em adultos com condições clínicas neurológicas que fazem uso de cadeira de rodas ${ }^{21}$. Algumas características como o delineamento, intervenção utilizada, duração da intervenção, amostra, métodos de avaliação e principais resultados, estão descritos na Tabela 1.

Devido ao número escasso de referências, optou-se por abordar os benefícios dos jogos de dança com um olhar multidisciplinar, avaliando os múltiplos benefícios proporcionados em diferentes tipos de doenças neurológicas, 


\section{que apesar de terem etiologias diferentes, apresentam}

\section{alguns déficits em comum.}

Tabela 1. Características dos estudos incluídos na revisão.

\begin{tabular}{|c|c|c|c|c|}
\hline Autores & Delineamento & $\begin{array}{c}\text { Intervenção, duração } \\
\text { e amostra }\end{array}$ & Métodos de avaliação & Resultados \\
\hline $\begin{array}{l}\text { Combs et al. } \\
(2012)^{18}\end{array}$ & $\begin{array}{l}\text { Coorte de } \\
\text { grupo único }\end{array}$ & $\begin{array}{l}\text { Jogo Hand Dance Pro }{ }^{\mathrm{TM}} \\
\text { em } 18 \text { sessões de } 30 \\
\text { minutos, durante } 6 \\
\text { semanas em } 10 \text { pessoas } \\
\text { com sequelas de } \\
\text { acidente vascular } \\
\text { cerebral. }\end{array}$ & $\begin{array}{l}\text { Análise tridimensional } \\
\text { do movimento da } \\
\text { extremidade superior } \\
\text { durante o alcance; } \\
\text { Wolf Motor Function } \\
\text { Test e a Escala de } \\
\text { impacto do AVC. }\end{array}$ & $\begin{array}{c}\text { Este estudo mostra que a } \\
\text { intervenção é viável e foi } \\
\text { capaz de causar melhorias } \\
\text { na cinemática de alcance } \\
\text { com o braço parético dos } \\
\text { participantes. }\end{array}$ \\
\hline $\begin{array}{l}\text { Hoang et al. } \\
(2015)^{7}\end{array}$ & $\begin{array}{c}\text { Ensaio } \\
\text { controlado } \\
\text { randomizado }\end{array}$ & $\begin{array}{l}\text { Sistema de treinamento } \\
\text { de passos desenvolvido } \\
\text { com o Stepmania e } \\
\text { utilizado por pelo menos } \\
\text { duas sessões de } \\
\text { treinamento de } 30 \\
\text { minutos por semana, } \\
\text { durante } 12 \text { semanas em } \\
50 \text { indivíduos com } \\
\text { esclerose múltipla. }\end{array}$ & $\begin{array}{l}\text { Tempo de reação de } \\
\text { passo de escolha } \\
\text { (CSRT); tempo do teste } \\
\text { de pisada stroop (SST); } \\
\text { Teste de equilíbrio } \\
\text { (oscilação postural, } \\
\text { componentes CSRT); } \\
\text { velocidade da marcha; } \\
\text { testes cognitivos; teste } \\
\text { de pino de nove } \\
\text { buracos ( } 9 \text {-HPT) e } \\
\text { escore composto } \\
\text { funcional ms (MSFC). }\end{array}$ & $\begin{array}{l}\text { Os autores observaram } \\
\text { que a intervenção se } \\
\text { mostrou segura e viável } \\
\text { em ambiente doméstico, } \\
\text { sem supervisão do } \\
\text { terapeuta. Além disso, foi } \\
\text { capaz de melhorar a } \\
\text { pisada, equilíbrio estático, } \\
\text { coordenação e } \\
\text { desempenho funcional dos } \\
\text { participantes. }\end{array}$ \\
\hline $\begin{array}{l}\text { Kloos et al. } \\
(2013)^{20}\end{array}$ & $\begin{array}{l}\text { Ensaio clínico } \\
\text { controlado }\end{array}$ & $\begin{array}{l}\text { Programa de exercícios } \\
\text { utilizando o Dance } \\
\text { Dance Revolution }{ }^{\mathrm{TM}} \text { por } \\
45 \text { minutos, } 2 \text { por } \\
\text { semana, durante } 6 \\
\text { semanas em } 18 \text { pessoas } \\
\text { com doença de } \\
\text { Huntington. }\end{array}$ & $\begin{array}{c}\text { Desempenho e } \\
\text { aderência ao jogo; } \\
\text { percepções dos } \\
\text { participantes do jogo; } \\
\text { segurança (sinais } \\
\text { vitais, alterações } \\
\text { adversas à saúde); } \\
\text { medidas espaço- } \\
\text { temporais da marcha; } \\
\text { Teste do Quatro- } \\
\text { Quadrado; Tinetti } \\
\text { Teste de Mobilidade; } \\
\text { Escala de Confiança de } \\
\text { Equilíbrio Específico } \\
\text { para Atividades; } \\
\text { Qualidade de Vida da } \\
\text { Organização Mundial de } \\
\text { Saúde - Bref. }\end{array}$ & $\begin{array}{c}\text { Neste estudo, pôde-se } \\
\text { observar que a } \\
\text { intervenção é segura e } \\
\text { motivadora para os } \\
\text { participantes e ainda } \\
\text { diminuiu o tempo em } \\
\text { suporte duplo durante a } \\
\text { caminhada, promovendo a } \\
\text { melhora do equilíbrio } \\
\text { dinâmico. }\end{array}$ \\
\hline $\begin{array}{l}\text { Lee; Lee e } \\
\text { Song. } \\
(2015)^{8}\end{array}$ & $\begin{array}{l}\text { Tipo de estudo } \\
\text { não descrito }\end{array}$ & $\begin{array}{c}\text { Tratamento } \\
\text { neurodesenvolvimento, } \\
\text { estimulação elétrica } \\
\text { funcional e exercício de } \\
\text { dança em realidade } \\
\text { virtual com o jogo } K \text {-pop } \\
\text { dance festival, realizado } \\
5 x \text { por semana, durante } \\
6 \text { semanas, em } 20 \\
\text { participantes com } \\
\text { Doença de Parkinson. }\end{array}$ & $\begin{array}{l}\text { Escala de equilíbrio de } \\
\text { Berg; Índice Modificado } \\
\text { de Barthel e o } \\
\text { Inventário de } \\
\text { Depressão de Beck. }\end{array}$ & $\begin{array}{l}\text { Os autores deste estudo } \\
\text { observaram a melhora do } \\
\text { equilíbrio, aumento da } \\
\text { independência durante as } \\
\text { atividades de vida diária } \\
\text { além de melhora do } \\
\text { estado depressivo. }\end{array}$ \\
\hline $\begin{array}{l}\text { Natbony et } \\
\text { al. }(2013)^{15}\end{array}$ & Série de casos & $\begin{array}{l}\text { Jogo Dance Dance } \\
\text { Revolution }{ }^{\mathrm{TM}} \text { em } 16 \\
\text { pessoas com Doença de } \\
\text { Parkinson leve a } \\
\text { moderada. }\end{array}$ & $\begin{array}{c}\text { Escala de Autoeficácia } \\
\text { para Exercício; Escala } \\
\text { de Equilíbrio e } \\
\text { Confiança específica } \\
\text { das atividades; técnica } \\
\text { nominal do grupo } \\
\text { (NGT). }\end{array}$ & $\begin{array}{c}\text { Esse estudo mostra que a } \\
\text { intervenção foi viável e } \\
\text { atraente. }\end{array}$ \\
\hline
\end{tabular}


Tabela 1 (cont.). Características dos estudos incluídos na revisão.

\begin{tabular}{|c|c|c|c|c|}
\hline Autores & Delineamento & $\begin{array}{c}\text { Intervenção, duração } \\
\text { e amostra }\end{array}$ & Métodos de avaliação & Resultados \\
\hline $\begin{array}{l}\text { Rowland e } \\
\text { Rimmer. } \\
(2012)^{21}\end{array}$ & Estudo de caso & $\begin{array}{l}\text { Três jogos de videogame } \\
\text { ativos (boliche wii, tênis } \\
\text { e uma versão adaptada } \\
\text { para extremidade } \\
\text { superior de um gamepad } \\
\text { Dance Dance } \\
\text { Revolution }{ }^{\mathrm{TM}} \text { ), } 10 \\
\text { minutos por atividade } \\
\text { realizados em dias } \\
\text { diferentes, em três } \\
\text { jovens adultos não } \\
\text { ambulantes. }\end{array}$ & $\begin{array}{l}\text { Calorimetria indireta e } \\
\text { Monitor de Frequência } \\
\text { Cardíaca Polar. }\end{array}$ & $\begin{array}{l}\text { Neste estudo foi } \\
\text { observado que a } \\
\text { adaptação desses jogos } \\
\text { para proporcionar o } \\
\text { aumento do gasto } \\
\text { energético foi viável. }\end{array}$ \\
\hline $\begin{array}{l}\text { Sampaio et } \\
\text { al. }(2016)^{19}\end{array}$ & Estudo de caso & $\begin{array}{l}\text { Treinamento de dança } \\
\text { aeróbica baseado em } \\
\text { realidade virtual, com o } \\
\text { jogo Just Dance } 3^{\text {TM }} \text { por } \\
1 \text { hora e } 25 \text { minutos a } 1 \\
\text { hora e } 40 \text { minutos com } \\
20 \text { sessões durante } 6 \\
\text { semanas em } 11 \\
\text { participantes com AVC } \\
\text { hemiparético. }\end{array}$ & $\begin{array}{l}\text { Monitor de frequência } \\
\text { cardíaca polar; } \\
\text { Teste de ergômetro de } \\
\text { ciclo submaximal da } \\
\text { YMCA; Pedômetro Tri- } \\
\text { Eixo Omran HJ-321. }\end{array}$ & $\begin{array}{l}\text { Neste estudo, observou-se } \\
\text { a melhora do controle } \\
\text { autônomo cardíaco após a } \\
\text { intervenção. }\end{array}$ \\
\hline $\begin{array}{l}\text { Song et al. } \\
(2017)^{16}\end{array}$ & $\begin{array}{l}\text { Ensaio clínico } \\
\text { randomizado } \\
\text { cego simples }\end{array}$ & $\begin{array}{c}\text { Treinamento domiciliar } \\
\text { mal supervisionado com } \\
\text { o jogo Stepmania por no } \\
\text { mínimo } 15 \text { minutos, } 3 \\
\text { vezes por semana, } \\
\text { durante } 12 \text { semanas em } \\
53 \text { pessoas com Doença } \\
\text { de Parkinson. }\end{array}$ & $\begin{array}{l}\text { Teste de tempo de } \\
\text { reação de escolha de } \\
\text { passos; Functional Gait } \\
\text { Assessment; Timed Up } \\
\text { and go; passarela } \\
\text { eletrônica; Avaliação } \\
\text { Cognitiva de Montreal; } \\
\text { teste de trilha; Novo } \\
\text { questionário de } \\
\text { congelamento da } \\
\text { marcha; Questionário } \\
\text { Internacional da Escala } \\
\text { de Eficácia de Queda. }\end{array}$ & $\begin{array}{c}\text { Os autores deste estudo } \\
\text { viram que a intervenção } \\
\text { não foi eficaz para } \\
\text { melhorar o desempenho } \\
\text { do passo, mobilidade, } \\
\text { potência muscular, } \\
\text { cognição, } \\
\text { tempo de reação simples, } \\
\text { congelamento da marcha, } \\
\text { medo de } \\
\text { cair, e redução do número } \\
\text { de quedas. }\end{array}$ \\
\hline $\begin{array}{l}\text { Sousa e } \\
\text { Bezerra. } \\
(2016)^{17}\end{array}$ & Série de casos & $\begin{array}{l}\text { Intervenção com uso de } \\
\text { tapete de vídeo dança } \\
\text { por } 45 \text { minutos em } 4 \\
\text { pessoas com Doença de } \\
\text { Parkinson. }\end{array}$ & $\begin{array}{l}\text { Teste de caminhada de } \\
10 \text { metros. }\end{array}$ & $\begin{array}{l}\text { Neste estudo foi observada } \\
\text { a redução do tempo para } \\
\text { percorrer um determinado } \\
\text { percurso. }\end{array}$ \\
\hline $\begin{array}{l}\text { Subramaniam } \\
\text { e Bhatt. } \\
(2019)^{9}\end{array}$ & $\begin{array}{l}\text { Estudo pré- } \\
\text { intervenção de } \\
\text { grupo único }\end{array}$ & $\begin{array}{c}\text { Jogo Just Dance } 3 \\
\text { durante } 6 \text { semanas, com } \\
20 \text { sessões, em } 13 \\
\text { pessoas com sequelas } \\
\text { de AVC. }\end{array}$ & $\begin{array}{c}\text { Eletromiografia de } \\
\text { superfície da atividade } \\
\text { muscular do ombro } \\
\text { durante a tarefa de } \\
\text { alcance em pé; } \\
\text { cinemática do ombro da } \\
\text { UE; escala de confiança } \\
\text { de equilíbrio específica } \\
\text { para atividades (ABC) e } \\
\text { o teste Timed-Up-and- } \\
\text { Go (TUG). }\end{array}$ & $\begin{array}{l}\text { Os autores deste estudo } \\
\text { observaram que o uso do } \\
\text { jogo promoveu melhorias } \\
\text { significativas no controle } \\
\text { do movimento do braço } \\
\text { parético e diminuiu o risco } \\
\text { de quedas. }\end{array}$ \\
\hline
\end{tabular}

\section{DISCUSSÃO}

Jogos que dependem do movimento corporal surgem como uma nova ferramenta para a prática de atividade física de forma gratificante, tendo como diferencial o estímulo competitivo e o desafio a ser superado no decorrer dos 
jogos $^{5,22}$ e apesar de não serem voltados para fins clínicos, podem ser usados na reabilitação, sendo tão eficaz quanto o tratamento convencional ${ }^{6}$.

Os jogos de dança mais comumente utilizados nos estudos encontrados foram o Dance Dance Revolution ${ }^{\text {TM }}$ (DDR), em que a interação do jogador com o jogo se dá por meio de um tapete ou plataforma ${ }^{23}$; Dance Central ${ }^{\mathrm{TM}}$ e Just Dance $^{\mathrm{Tm}}$, que necessitam do movimento de todo o corpo para se obter resultados durante a experiência no jogo 22 ; e a versão modificada do DDR, o Stepmania (www.stepmania.com) que apresenta como vantagem o seu código aberto. Este jogo permite que sejam feitas modificações em seu software, assim torna-se mais fácil a adaptação dos parâmetros para o uso na reabilitação ${ }^{2}$. Todos estes jogos sincronizam os passos de dança com as músicas escolhidas pelo usuário e torna a experiência mais agradável.

Videogames comerciais vêm se mostrando efetivos quando utilizados em integração com a reabilitação convencional ${ }^{6}$, entretanto não são voltados às metas clínicas específicas, e possuem design infantil ${ }^{12}$. Apesar disso, estudos mostram que os jogos de dança são vistos como agradáveis, além de indicar bons níveis de adesão por parte de idosos e pessoas com doenças neurológicas ${ }^{2,15,20,24}$.

Em um estudo de série de casos de casos foi observada a percepção referente a um programa de exercícios com uso do DDR através do feedback de 16 participantes com doença de Parkinson. Algumas das vantagens mais relatadas foram a facilidade no uso, diversão, melhora de equilíbrio e 
coordenação, além de perceberem como um exercício aeróbico completo e desafiador, sugerindo que o DDR é bem tolerado, atrativo e reparado como benéfico em indivíduos com doença de Parkinson. Entretanto, preocupações com o custo, possíveis problemas técnicos e relatos de interface confusa também foram observados neste estudo ${ }^{15}$.

Dificuldades por parte dos idosos em lidar com novas tecnologias também podem servir como barreira para o uso do videogame ${ }^{2}$ e pessoas com limitações físicas mais graves podem não conseguir jogar adequadamente sendo sugestivo o uso por pessoas com deficiência leve a moderada7,20. Entretanto, modificações podem ser feitas em alguns jogos, possibilitando a inclusão de pessoas que fazem uso de cadeira de rodas.

Estudos mostram que jogos de dança que utilizam tapetes, podem ser adaptados com um dispositivo de controle manual para pessoas não ambulantes e com diferentes níveis de comprometimento neurológico ${ }^{18,21}$, e assim consigam usufruir de alguns benefícios de um jogo de dança como o aumento do gasto energético ${ }^{21}$ e melhorias no padrão de movimento dos membros superiores ${ }^{18}$.

Uma possibilidade para os jogos de dança é o uso em ambiente domiciliar, no qual possui a vantagem de o jogador ter apoio da família e não precisar sair de sua residência, diminuindo as chances de afastamento do processo terapêutico. Estudos que realizaram esse tipo de treinamento em ambiente domiciliar mostraram que os jogos de dança são viáveis e seguros de serem utilizados por 
pessoas com deficiências moderadas, com supervisão através de telefonemas e orientações escritas ${ }^{7}$, e que participantes como deficiências mais graves sentem mais dificuldade durante o uso dos videogames e necessitam de supervisão direta de um fisioterapeuta ou pessoa treinada para evitar episódios de quedas ${ }^{16,20}$. Portanto, faz-se necessária a avaliação de um fisioterapeuta para identificar as habilidades físicas do jogador e ajudar na seleção das músicas adequando a velocidade delas antes de se iniciar o jogo 20 .

Sobre o tempo e frequência do uso dos jogos de dança na reabilitação neurológica, os estudos encontrados mostram benefícios com diferentes parâmetros utilizados. Um estudo evidenciou que mesmo com apenas uma única intervenção de uma hora, mudanças significativas são observadas na redução do tempo para se percorrer um

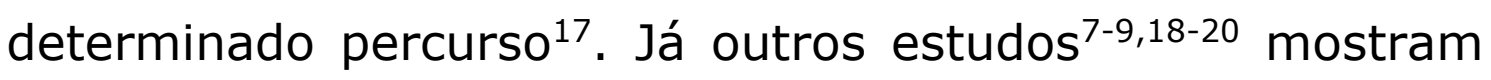
uma maior quantidade de melhorias com intervenções realizadas por no mínimo 30 minutos semanais, totalizando 12 sessões. Entretanto, um estudo que utilizou 0 treinamento de passos domiciliares com o Stepmania em pessoas com Doença de Parkinson leve a moderada por no mínimo 15 minutos, 3 vezes por semana, durante 12 semanas, não obteve resultados significativos e isso foi atribuído à baixa dosagem do exercício ${ }^{16}$. Então observa-se que pessoas com problemas neurológicos podem necessitar de um tempo diário de intervenção maior para se ter uma dosagem adequada ${ }^{16}$. 


\section{Benefícios físicos, cognitivo-motores e psicossociais}

Sabe-se que a prática de atividades físicas favorece a neuroplasticidade cerebral em indivíduos com distúrbio neurológico ou declínio cognitivo através do aumento da produção de fatores neurotróficos que promovem 0 crescimento dendrítico, neurogênese e potência neuronal ${ }^{25}$. Quando associados à música, os exercícios podem ter seus benefícios otimizados. Além da música ter a capacidade de ativar o cérebro de uma forma quase global e poder estimular a neuroplasticidade, ela também possui a capacidade de facilitar a execução e melhorar a qualidade do movimento corporal ${ }^{26}$.

Diversas doenças neurológicas podem ser acompanhadas de declínio cognitivo, assim como no AVC, em que 4 a cada 10 pessoas apresentam comprometimento cognitivo (ainda não considerada demência) no primeiro ano após o acidente vascular ${ }^{27}$.

Intervenções baseadas em dança têm o potencial de proporcionar melhorias nas estruturas e funções cerebrais, e podem fortalecer a conexão de diferentes áreas sensoriais e cognitivas promovendo benefícios globais como a melhora da memória, atenção, cognição, equilíbrio, e de fatores psicossociais $^{28}$.

Os jogos interativos de dança trazem a possibilidade de associar o exercício aeróbico com o treinamento cognitivo, através da observação das setas ou do movimento feito pelo avatar no jogo, assim, obtém-se pistas visuais para o planejamento de estratégias de movimento ao mesmo 
tempo em que há a sincronização dos passos com a música ${ }^{29}$.

A combinação de componentes físicos e cognitivos com jogos de dança é capaz de reduzir a atividade da região préfrontal no cérebro durante a caminhada, indicando o aumento da eficiência, aperfeiçoamento da função executiva e da velocidade de processamento dessa região ${ }^{29}$.

Em um estudo que realizou uma única intervenção utilizando jogo com tapete de dança foram evidenciados benefícios na marcha de pacientes com Doença de Parkinson. Este resultado está relacionado à estimulação de funções cognitivo-motoras como o planejamento motor e funções executivas, favorecendo a realização da marcha ${ }^{17}$. Assim como outro ensaio clínico que encontrou melhorias na marcha em pessoas com Esclerose Múltipla utilizando o jogo Stepmania e propôs os mesmos mecanismos relatados anteriormente para explicação do seu?. Além disso, estudos mostram que as intervenções de dança propiciam bons resultados relacionados à cognição global, velocidade da marcha, atividades em dupla tarefa e mobilidade funcional em indivíduos com Doença de Parkinson, favorecendo maior independência e autonomia dessas pessoas ${ }^{4,10}$.

O uso dos jogos de dança pode ser útil para diminuição do risco de quedas em pessoas com doenças neurológicas $7,9,17$ e alguns fatores, como a melhora do equilíbrio, contribuem para isso. Sugere-se que a dança pode melhorar o equilíbrio e a marcha em adultos com doenças neurológicas ${ }^{3}$. 
Outros estudos realizados com indivíduos com Esclerose Múltipla, Doença de Huntington, AVC e Doença de Parkinson também demonstraram a melhora do equilíbrio após intervenção com jogos de dança7,8,20,30. Este ganho de equilíbrio pode ser explicado pela realização de complexos movimentos corporais requeridos em um jogo de dança, no qual exigem o controle postural antecipatório para que a estabilidade seja mantida durante o deslocamento do próprio peso em diferentes direções e para se preparar para as próximas sequências de movimentos da coreografia ${ }^{30}$.

Outros tipos de benefícios físicos também foram encontrados em um estudo que utilizou o jogo Dance Central ${ }^{\mathrm{TM}}$ com Kinect em pessoas idosas. Observou-se 0 aumento do pico de torque excêntrico dos músculos isquiotibiais, que pode ser atribuída ao tipo de protocolo utilizado, no qual houve a inserção de elementos para progressão da dificuldade do exercício, como solo instável e perturbação visual, contribuindo assim para a melhora de aspectos musculoesqueléticos ${ }^{24}$.

Além dos benefícios cognitivo-motores e musculoesqueléticos, são encontrados também efeitos positivos no condicionamento cardiovascular. Em um estudo que investigou os ajustes da modulação e da frequência cardíaca autônoma em 11 indivíduos com sequelas do AVC, após intervenção com o jogo Just Dance $3^{\mathrm{TM}}$, foi observada a melhora do tom vagal (atividade do nervo vago) e da variabilidade cardíaca ${ }^{19}$. 
Alterações físicas/motoras são bem frequentes em condições clínicas decorrentes de doenças neurológicas ${ }^{3}$, entretanto, regularmente também são observadas alterações psicossociais, como sintomas de ansiedade e de depressão. Em indivíduos com Esclerose Múltipla a prevalência desses sintomas pode chegar a 35\% (sintomas depressivos) e 34\% (sintomas de ansiedade) e podem interferir negativamente no desenvolvimento da doença ${ }^{31}$.

Em um ensaio clínico realizado com indivíduos com Doença de Parkinson, foi demonstrado que um jogo de dança é capaz de causar melhorias no estado de transtorno depressivo ${ }^{8}$. Estudos mostram que os exergames baseados em dança contribuíram para a redução significativa do estado de ansiedade e de sintomas depressivos em indivíduos sem disfunções neurológicas ${ }^{24,32}$.

A dança também tem o potencial de influenciar o estado emocional e as interações sociais em indivíduos com Alzheimer $^{33}$. Intervenções de dança que promovam a interação social podem contribuir para maior participação das pessoas em atividades deste tipo ${ }^{34}$.

\section{CONCLUSÃO}

Os jogos de dança se apresentam como um recurso acessível e divertido, que traz possibilidades de adaptação para ser implantado em ambulatórios ou utilizados em ambiente domiciliar de forma segura.

O uso dos jogos de dança é promissor em adultos e idosos com condições neurológicas como a Doença de 
Parkinson, Doença de Huntington, Esclerose Múltipla e AVC e apesar dos achados serem limitados, eles apontam para um grande potencial na obtenção de diversos benefícios físicos, cognitivos e psicossociais percebidos com a utilização desses jogos. Entretanto, novos estudos são necessários para maior exploração sobre essa temática e para maiores esclarecimentos sobre as melhorias proporcionadas.

\section{REFERÊNCIAS}

1.Silva KM, Nitchske RG, Santos SM. A dança e o envelhecimento: benefícios descritos na literatura. Cienc Cuidado Saúde 2018;17:1-7. https://doi.org/10.4025/cienccuidsaude.v17i3.39020

2.Schoene D, Lord SR, Delbaere K, Severino C, Davies TA, Smith ST. A randomized controlled pilot study of home-based step training in older people using videogame technology. PLoS One 2013;8:e57734. https://doi.org/10.1371/journal.pone.0057734

3.Patterson KK, Wong JS, Prout EC, Brooks D. Dance for the rehabilitation of balance and gait in adults with neurological conditions other than Parkinson's disease: A systematic review. Heliyon 2018;4:e00584. https://doi.org/10.1016/j.heliyon.2018.e00584

4. Kalyani HH, Sullivan K, Moyle G, Brauer S, Jeffrey ER, Roeder L, et al. Effects of Dance on Gait, Cognition, and Dual-Tasking in Parkinson's Disease: A Systematic Review and Meta-Analysis. J Parkinsons Dis 2019;9:335-49. https://doi.org/10.3233/JPD-181516

5. Muñoz JE, Villada JF, Carlos J, Trujillo G. Exergames: a technological tool for the physical activity. Rev Med Risaralda 2013;19:126-30. http://www.scielo.org.co/pdf/rmri/v19n2/v19n2a05.pdf

6 . Bonnechère $B$, Jansen $B$, Omelina $L$, Van Sint Jan $S$. The use of commercial video games in rehabilitation: a systematic review. Int J Rehabil Res 2016;39:277-90.

https://doi.org/10.1097/MRR.0000000000000190

7. Hoang P, Schoene D, Gandevia S, Smith S, Lord SR. Effects of a home-based step training programme on balance, stepping, cognition and functional performance in people with multiple sclerosis: a randomized controlled trial. Mult Scler 2016;22:94-103. https://doi.org/10.1177/1352458515579442

8. Lee NY, Lee DK, Song HS. Effect of virtual reality dance exercise on the balance, activities of daily living, and depressive disorder status of Parkinson's disease patients. J Phys Ther Sci 2015;27:1457. https://doi.org/10.1589/jpts.27.145

9.Subramaniam S, Bhatt T. Dance-based exergaming for upper extremity rehabilitation and reducing fall-risk in community-dwelling 
individuals with chronic stroke. A preliminary study. Top Stroke Rehabil 2019;26:565-75. https://doi.org/10.1080/10749357.2019.1625545 10.Delabary MS, Komeroski IG, Monteiro EP, Costa RR, Haas AN. Effects of dance practice on functional mobility, motor symptoms and quality of life in people with Parkinson's disease: a systematic review with meta-analysis. Aging Clin Exp Res 2018;30:727-35. https://doi.org/10.1007/s40520-017-0836-2

11.Zhang Q, Hu J, Wei L, Jia Y, Jin Y. Effects of dance therapy on cognitive and mood symptoms in people with Parkinson's disease: A systematic review and meta-analysis. Complement Ther Clin Pract 2019;36:12-7. https://doi.org/10.1016/j.ctcp.2019.04.005

12.Perrochon A, Borel B, Istrate D, Compagnat M, Daviet JC. Exercisebased games interventions at home in individuals with a neurological disease: A systematic review and meta-analysis. Ann Phys Rehabil Med 2019;62:366-78. https://doi.org/10.1016/j.rehab.2019.04.004

13. Mura G, Carta MG, Sancassiani F, Machado S, Prosperini L. Active exergames to improve cognitive functioning in neurological disabilities: a systematic review and meta-analysis. Eur J Phys Rehabil Med 2018;54:450-62. https://doi.org/10.23736/S1973-9087.17.04680-9

14. Rosly MM, Rosly HM, Oam GM, Husain R, Hasnan N. Exergaming for individuals with neurological disability: a systematic review. Disabil Rehabil 2017;39:727-35.

https://doi.org/10.3109/09638288.2016.1161086

15. Natbony LR, Zimmer A, Ivanco LS, Studenski SA, Jain S. Perceptions of a Videogame-Based Dance Exercise Program Among Individuals with Parkinson's Disease. Games Health J 2013;2:235-9. https://doi.org/10.1089/g4h.2013.0011

16.Song J, Paul SS, Caetano MJD, Smith S, Dibble LE, Love R, et al. Home-based step training using videogame technology in people with Parkinson's disease: a single-blinded randomised controlled trial. Clin Rehabil

https://doi.org/10.1177/0269215517721593

17.Sousa AS, Bezerra PP. A realidade virtual por meio do tapete de videodança melhora a marcha de pacientes com doença de Parkinson. Rev Bras Neurol 2016;52:21-9.

https://revistas.ufrj.br/index.php/rbn/article/view/3201

18.Combs SA, Finley MA, Henss M, Himmler S, Lapota K, Stillwell D. Effects of a repetitive gaming intervention on upper extremity impairments and function in persons with chronic stroke: a preliminary study. Disabil Rehabil 2012;34:1291-8. https://doi.org/10.3109/09638288.2011.641660

19.Sampaio LM, Subramaniam S, Arena R, Bhatt T. Does Virtual Reality-based Kinect Dance Training Paradigm Improve Autonomic Nervous System Modulation in Individuals with Chronic Stroke? J Vasc Interv Neurol 2016;9:21-9.

https://www.ncbi.nlm.nih.gov/pmc/articles/PMC5094257/pdf/jvin-92-5.pdf

20.Kloos AD, Fritz NE, Kostyk SK, Young GS, Kegelmeyer DA. Video game play (Dance Dance Revolution) as a potential exercise therapy in 
Huntington's disease: a controlled clinical trial. Clin Rehabil 2013;27:972-82. https://doi.org/10.1177/0269215513487235

21.Rowland JL, Rimmer JH. Feasibility of using active video gaming as a means for increasing energy expenditure in three nonambulatory young adults with disabilities. PM R 2012;4:569-73. https://doi.org/10.1016/j.pmrj.2012.03.011

22. Bronner S, Pinsker R, Noah JA. Energy cost and game flow of 5 exer-games in trained players. Am J Health Behav 2013;37:369-80. https://doi.org/10.5993/AJHB.37.3.10

23.Prudente PLG. A Dança na Perspectiva de um Jogo Digital. Licere 2018;21:386-409. https://doi.org/10.35699/1981-3171.2018.1874

24.Rodrigues EV, Gallo LH, Guimarães ATB, Melo Filho J, Luna BC, Gomes ARS. Effects of Dance Exergaming on Depressive Symptoms, Fear of Falling, and Musculoskeletal Function in Fallers and Nonfallers Community-Dwelling Older Women. Rejuvenation Res 2018;21:51826. https://doi.org/10.1089/rej.2017.2041

25. Mackay CP, Kuys SS, Brauer SG. The Effect of Aerobic Exercise on Brain-Derived Neurotrophic Factor in People with Neurological Disorders: A Systematic Review and Meta-Analysis. Neural Plast 2017;2017:4716197. https://doi.org/10.1155/2017/4716197

26.Jurado-Noboa C. La Musicoterapia Neurológica Como Modelo de Neurorrehabilitación. Neurologic Music Therapy As A Neurorehabilitation Model. Rev Ecuat Neurol 2018;27:72-9. http://scielo.senescyt.gob.ec/pdf/rneuro/v27n1/2631-2581-rneuro27-01-00072.pdf

27.Sexton E, McLoughlin A, Williams DJ, Merriman NA, Donnelly N, Rohde $D$, et al. Systematic review and meta-analysis of the prevalence of cognitive impairment no dementia in the first year post-stroke. Eur Stroke J 2019;4:160-71.

https://doi.org/10.1177/2396987318825484

28. Teixeira-Machado L, Arida RM, de Jesus Mari J. Dance for neuroplasticity: A descriptive systematic review. Neurosci Biobehav Rev 2019;96:232-40.

https://doi.org/10.1016/j.neubiorev.2018.12.010

29.Eggenberger $P$, Wolf $M$, Schumann $M$, de Bruin ED. Exergame and Balance Training Modulate Prefrontal Brain Activity during Walking and Enhance Executive Function in Older Adults. Front Aging Neurosci 2016;8:66. https://doi.org/10.3389/fnagi.2016.00066

30.Subramaniam S, Bhatt T. Does A Virtual Reality-Based Dance Training Paradigm Increase Balance Control in Chronic Stroke Survivors? A Preliminary Study. Int J Neurorehabilitation 2015;4:1-10. https://doi.org/10.4172/2376-0281.1000185

31.Boeschoten RE, Braamse AM, Beekman AT, Cuijpers P, van Oppen $\mathrm{P}$, Dekker J, et al. Prevalence of depression and anxiety in Multiple Sclerosis: A systematic review and meta-analysis. J Neurol Sci 2017;372:331-41. https://doi.org/10.1016/j.jns.2016.11.067

32.Viana RB, Alves CL, Vieira CA, Vancini RL, Campos $M H$, Gentil $P$, et al. Anxiolytic effects of a single session of the exergame Zumba ${ }^{\circledR}$ 
Fitness on healthy young women. Games Health $] 2017 ; 6: 365-70$. https://doi.org/10.1089/g4h.2017.0085

33.Ruiz-Muelle A, López-Rodríguez MM. Dance for People with Alzheimer's Disease: A Systematic Review. Curr Alzheimer Res 2019;16:919-33.

https://doi.org/10.2174/1567205016666190725151614

34.Bronner S, Pinsker R, Naik R, Noah JA. Physiological and psychophysiological responses to an exer-game training protocol. J Sci Med Sport 2016;19:267-71.

https://doi.org/10.1016/j.pmrj.2012.03.011 